\title{
Draft Genome of the Edible Oriental Insect Protaetia brevitarsis seulensis
}

\author{
Joon Ha Lee ${ }^{1 t}$, Myunghee Jung ${ }^{2 t}$, Younhee Shin ${ }^{2}$, Sathiyamoorthy Subramaniyam ${ }^{2}$, \\ In-Woo Kim ${ }^{1}$, Minchul Seo ${ }^{1}$, Mi-Ae Kim ${ }^{1}$, Seong Hyun Kim ${ }^{1}$, Jihye Hwang ${ }^{3}$, \\ Eun Hwa Choi ${ }^{3}$, Ui Wook Hwang ${ }^{3}$ and Jae Sam Hwang ${ }^{1 *}$
}

${ }^{1}$ Department of Agricultural Biology, National Institute of Agricultural Sciences, Rural Development Administration, Wanju, South Korea, ${ }^{2}$ Research and Development Center, Insilicogen Inc., Yongin, South Korea, ${ }^{3}$ Department of Biology Education, Teachers College and Institute for Phylogenomics and Evolution, Kyungpook National University, Daegu, South Korea

Keywords: Cetoniinae, Kolbe, genome, Protaetia brevitarsis seulensis, edible insect

\section{OPEN ACCESS}

Edited by:

Xianyao $L i$

Shandong Agricultural

University, China

Reviewed by:

Murukarthick Jayakodi, Leibniz Institute of Plant Genetics and Crop Plant Research (IPK), Germany

Byungwook Lee,

Korea Research Institute of

Bioscience and Biotechnology

(KRIBB), South Korea

${ }^{*}$ Correspondence:

Jae Sam Hwang

hwangjs@korea.kr

†These authors have contributed equally to this work

Specialty section:

This article was submitted to

Livestock Genomics,

a section of the journal

Frontiers in Genetics

Received: 12 August 2020 Accepted: 10 December 2020

Published: 13 January 2021

Citation:

Lee $J H$, Jung $M$, Shin $Y$, Subramaniyam S, Kim I-W, Seo M, Kim M-A, Kim SH, Hwang J, Choi EH,

Hwang UW and Hwang JS (2021) Draft Genome of the Edible Oriental Insect Protaetia brevitarsis seulensis.

Front. Genet. 11:593994.

doi: 10.3389/fgene.2020.593994

\section{INTRODUCTION}

Insects hold the template for significant technological and biological inventions, since most of them are smaller in size and have different characteristics. It could help human lives, if scientists mimic their characteristics for sensors, robotics, agriculture, and medicine. Recently, insects were identified as an alternative source for meat to meet the Food and Agricultural Organization (FAO) food demand for the growing population, which is estimated to be 9 billion by 2050 (Han et al., 2017). The recent progress and research interest in the field of entomophagy explain the importance of insect breeding (Raheem et al., 2019). In parallel, the inherited problem in the selection of insects for breeding is also harmful to the environment. To cite an example, Locust, a grasshopper group rich in nutrients and protein content, can be utilized as a substitute for meat, but it is highly harmful to the environment and food crops worldwide (Le Gall et al., 2019). Hence, it is essential to carefully adapt an insect from the indigenous population around the world, i.e., people who consume insects in their regular diet for various reasons. Moreover, insect breeding is estimated to reduce $\mathrm{CO}_{2}$ emission in the atmosphere (i.e., up to $18 \%$ ) when compared to animal breeding, which is as crucial as food production (Raheem et al., 2019). Other major drawbacks of insect-based foods are toxicities and allergens, which need to be eliminated through detailed characterizations. By considering all these factors, the genetic make-up was initiated through a large insect genome project to fuel detailed characterizations (i.e., i5K insect genomes). However, for various reasons, the project has not reached the desired goal so far (Li et al., 2019a). Furthermore, the highestsequenced species in i5k belong to the Coleoptera taxonomical order, which has more edible insects with beneficial medicinal and agricultural importance.

In South Korea, the estimated market value for edible insects in 2020 is USD 457 million (Han et al., 2017). Notably, the white-spotted flower chafer beetle has contributed to the highest revenue among other edible insects. As per the Korean Ministry of Agriculture, Food and Rural Affairs (https://www.mafra.go.kr/english/1412/subview.do) report, the insect breeding industries rose from 726 in 2015 to 2,318 ( 300\%) in 2018. Based on this knowledge, we selected the oriental edible beetle insect, Protaetia brevitarsis seulensis, also known as Kolbe, for genome sequencing (referred to as Kolbe in the rest of the article). Kolbe belongs to the Cetoniinae family, widely used in oriental medicine to treat various diseases. Also, it was approved temporarily as a food material by the Ministry of Food and Drug Safety of Korea (MFDS) in 2014 (Lee et al., 2017b). It has been highly suggested for use in cookies and cosmetics (Lee et al., 2017a). Therapeutic components such as phenols (Kim et al., 2020), alkaloids (Lee et al., 2017a), fatty acids (Li et al., 2019b), and bioactive peptides (Lee et al., 2016) were characterized from this species to treat different diseases. In agriculture, the waste management process, such as livestock manure processing (Yin et al., 2018) and plant cellulose decomposition, uses Kolbe (Li et al., 2019b). However, in the genus Protaetia, 
there are only two species, namely, Protaetia brevitarsis and Kolbe. The draft genome of Protaetia brevitarsis habituated in China, as reported so far, is heterozygous. But as per our knowledge, this is the first draft genome of the species Kolbe widely present in South Korea.

\section{Value of the Data}

The Kolbe draft genome is a base/reference for all the molecular studies in the Protaetia genus. It could be a valuable resource to conduct a comparative analysis among the species in the genome of the Protaetia genus to enhance breeding.

\section{MATERIALS AND METHODS}

\section{Insect Sample Collection}

Kolbe was maintained in the insect rearing facility of the National Institute of Agricultural Sciences (Wanju, Republic of Korea). The larvae and adults were reared on fermented oak sawdust in a constant rearing room at $25^{\circ} \mathrm{C} \pm 1^{\circ} \mathrm{C}$, under $50-60 \%$ relative humidity $(\mathrm{RH})$ and a $14 \mathrm{~h}$ light: $10 \mathrm{~h}$ dark photoperiod cycle.

\section{DNA and RNA Preparation for Sequencing}

Eighteen individual last instar larvae of Kolbe were selected for DNA sample extraction from the whole body for the genomic sequencing. For the genomic DNA isolation, the sample was washed with PBS, sterilized with $70 \%$ ethanol, and then anesthetized on ice. The entire body was fixed, and the dissected integument was then cut along the ventral part, and the guts were removed. The carcass was then quickly ground in liquid nitrogen using a mortar and pestle. The ground tissues were used for genomic DNA isolation using a Wizard Genomic DNA Purification Kit (Promega, USA) according to the manufacturer's instruction. The quality and quantity of the DNA sample were examined using ultraviolet (UV) absorbance and gel electrophoreses. Additionally, total RNA from four different tissues (fat body, gut, muscle, and hemocytes) and four different developmental stages (egg, larva, pupa, and adult) were isolated for whole transcriptome sequencing. Briefly, each tissue (fat body, gut, and muscle) was collected from three individual last instar larvae after dissection, as mentioned above. For the collection of hemocytes, three individual last instar larval hemolymph were directly collected into sterile tubes containing anticoagulant buffer $(62 \mathrm{mM} \mathrm{NaCl}, 100 \mathrm{mM}$ glucose, $10 \mathrm{mM}$ EDTA, $30 \mathrm{mM}$ Sodium citrate, $26 \mathrm{mM}$ citric acid, and $\mathrm{pH} 4.6$ ) on ice in triplicate, and they were centrifuged for $10 \mathrm{~min}$ at $1,000 \mathrm{~g}$ at $4{ }^{\circ} \mathrm{C}$ to remove the supernatant. The tissues were homogenized in a $1.5 \mathrm{ml}$ tube containing TRIzol reagent (Invitrogen, Carlsbad, CA, USA) using a pestle. In the case of the developmental stage sample, each stage of the three individual samples was washed with $70 \%$ ethanol to reduce microbial contamination from its
A

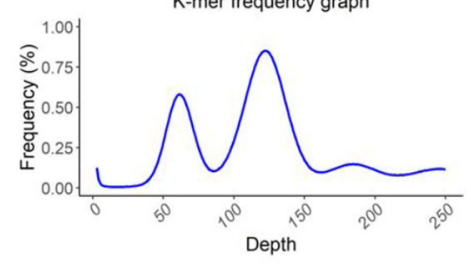

B

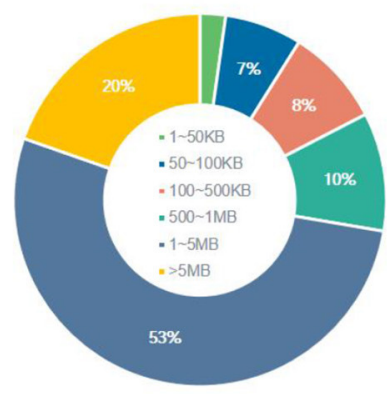

C

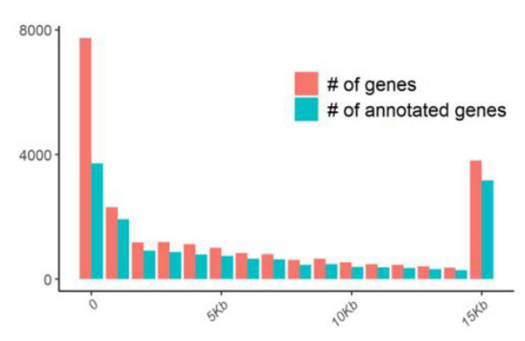

D

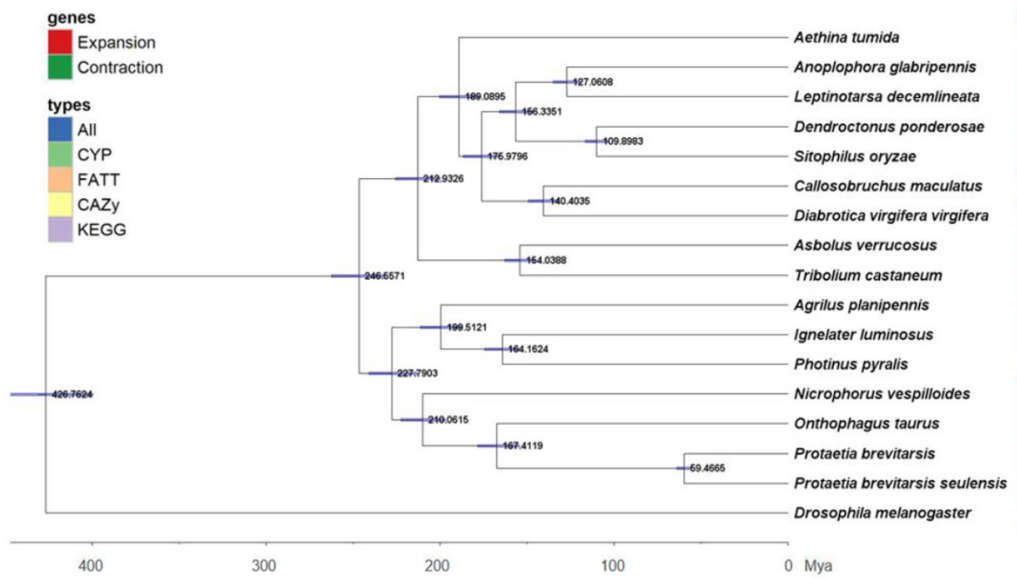

\begin{tabular}{|c|c|c|c|c|c|c|c|c|c|}
\hline \hline 1026 & -8493 & 2 & -39 & 22 & -108 & 25 & -186 & 21 & -92 \\
\hline 758 & -2532 & 6 & -11 & 26 & -39 & 27 & -56 & 19 & -40 \\
\hline 617 & -2889 & 3 & -10 & 12 & -66 & 10 & -77 & 15 & -39 \\
\hline 737 & -2637 & 4 & -13 & 10 & -41 & 17 & -61 & 12 & -44 \\
\hline 801 & -2281 & 2 & -16 & 11 & -50 & 20 & -78 & 12 & -45 \\
\hline 3617 & -3631 & 4 & -12 & 47 & -66 & 56 & -85 & 46 & -44 \\
\hline 1407 & -3793 & 6 & -6 & 16 & -46 & 20 & -64 & 19 & -55 \\
\hline 648 & -2691 & 2 & -31 & 14 & -94 & 11 & -95 & 12 & -41 \\
\hline 427 & -2464 & 6 & -8 & 9 & -44 & 16 & -39 & 13 & -35 \\
\hline 827 & -6157 & 3 & -18 & 21 & -82 & 26 & -96 & 13 & -64 \\
\hline 1864 & -3397 & 6 & -10 & 23 & -44 & 24 & -63 & 20 & -50 \\
\hline 2264 & -3598 & 7 & -10 & 31 & -54 & 41 & -52 & 34 & -40 \\
\hline 433 & -6777 & 0 & -23 & 11 & -109 & 10 & -94 & 14 & -91 \\
\hline 843 & -5687 & 7 & -10 & 20 & -74 & 20 & -87 & 12 & -85 \\
\hline 1353 & -2929 & 3 & -7 & 19 & -60 & 17 & -63 & 21 & -51 \\
\hline 880 & -2810 & 1 & -10 & 9 & -58 & 15 & -56 & 14 & -66 \\
\hline 545 & 1749 & 1 & -10 & 3 & -358 & 8 & -274 & 16 & -151 \\
\hline
\end{tabular}

FIGURE 1 | Summary of the sequencing; (A) Genome size estimation, (B) Contig length distribution, (C) Genes with annotation overview, (D) Gene expansion and contraction among the insect Coleoptera order genomes along with the phylogenetic tree reconstructed by BEAST with single-copy genes. 
surface. After ethanol volatilization, the individual was then quickly ground into a fine powder in liquid nitrogen, except for the eggs. For the extraction of eggs, 10 eggs were homogenized in a $1.5 \mathrm{ml}$ tube containing TRIzol reagent using a pestle, in triplicate. RNA quantitation was performed by UV absorbance, and gel electrophoreses further confirmed its quality.

\section{Genome Size Estimation and Assembly}

The isolated DNAs were sequenced using two different sequencing methods, i.e., Pacific bioscience (PacBio, Sequel), and Illumina (NextSeq500), which is familiar for long and short read sequencing. DNALink, the authorized service provider in South Korea, conducted complete experimental procedures. The Illumina paired-end sequences were initially subjected to the filtering of technical artifacts (i.e., base calling error [PHERD quality score $(Q \leq 20)]$, and adapters using Trimmomatic0.32 method (Bolger et al., 2014). Finally, the genome size estimation was carried out using the $k$-mer-based method with the Jellyfish v2.0 by calculating the genome coverage depth and size, as explained in the Sea Bream genome article (Shin et al., 2018). Additionally, these Illumina reads were used for the error correction of PacBio reads with clc-assembly-cell v5.1.1.184548-201811011136. Finally, the corrected PacBio reads were used for the initial draft version of the Kolbe genome with FALCON-Unzip v0.30 and haplotype assembler (Chin et al., 2016). The assembled contigs were assessed for completeness using the BUSCO v3.0, with the insecta_odb9 reference datasets (Waterhouse et al., 2017).

\section{REPEAT REGIONS PREDICTION AND CLASSIFICATION}

The repeat regions in Kolbe were predicted using RepeatModeler (www.repeatmasker.org/RepeatModeler/) and classified into subclasses with the reference Repbase v20.08 database (www.girinst.org/repbase/) (Bao et al., 2015). Finally, the repeats were masked in the genome using RepeatMasker v4.0.5 (www.repeatmasker.org) with RMBlastn v2.2.27+.

\section{GENE PREDICTION AND ANNOTATION}

The genes from the Kolbe draft were predicted using an inhouse gene prediction pipeline. It includes three modules: an evidence-based gene modeler (EVM), an ab-initio gene modeler, and a consensus gene modeler. The transcriptomes from the two methods [i.e., Illumina $(132.8 \mathrm{~Gb})$ and IsoSeq $(0.7 \mathrm{~Gb})]$ were mapped to the Kolbe repeat masked draft genome using TopHat, and Cufflink (Trapnell et al., 2012) and PASA (Haas et al., 2003) marked the transcripts and gene structural boundaries respectively. The ab-initio gene modeler and EVM (includes Exonerate (Slater and Birney, 2005), AUGUSTUS (Stanke et al., 2006), and GENEID (Blanco et al., 2002)) were trained with several genomes. The final gene and transcript models were optimized with a consensus gene modeler with EVidenceModeler (Haas et al., 2008). The functional annotations (i.e., gene ontologies (GO), KEGG Pathways) for
TABLE 1 | Summary of the sequencing till annotation of Protaetia brevitarsis seulensis draft genome.

\begin{tabular}{|c|c|c|}
\hline Types & PacBio(Gb) & Illumina(Gb) \\
\hline \multicolumn{3}{|l|}{ (A) SEQUENCING } \\
\hline DNA & 31.1 & 277.7 \\
\hline RNA & 0.7 & 132.8 \\
\hline \multicolumn{3}{|l|}{ (B) ASSEMBLY } \\
\hline Estimated genome size (bp) & $656,797,776$ & \\
\hline Contigs & 224 & \\
\hline Contig length (bp) & $692,712,625$ & \\
\hline Average length (bp) & $3,092,467.08$ & \\
\hline Minimum length (bp) & 26,261 & \\
\hline Maximum length (bp) & $16,895,244$ & \\
\hline N50 (bp) & $4,997,170$ & \\
\hline NG50 (bp) & $5,158,302$ & \\
\hline $\mathrm{N}(\%)$ & 0 & \\
\hline GC (\%) & 33.42 & \\
\hline Repeat (\%) & $344,334,720(49.71 \%)$ & \\
\hline BUSCO (insecta) complete (\%) & 99.03 & \\
\hline \multicolumn{3}{|l|}{ (C) STRUCTURAL ANNOTATIONS } \\
\hline \# of genes & 23,551 & \\
\hline Average gene length (bp) & $8,217.32$ & \\
\hline Gene coverage (\%) & $193,526,041$ (27.94\%) & \\
\hline GC in CDS (\%) & 43.67 & \\
\hline Exon/Gene & 3.97 & \\
\hline Average exon length (bp) & 250.25 & \\
\hline Exon coverage (\%) & 23,416,675 (3.38\%) & \\
\hline Average intron length (bp) & $2,429.34$ & \\
\hline Intron coverage (\%) & $170,109,366$ (24.56\%) & \\
\hline \multicolumn{3}{|l|}{ (D) FUNCTIONAL ANNOTATIONS } \\
\hline No hits & 7,884 (33.48\%) & \\
\hline Blast hits & $15,667(66.52 \%)$ & \\
\hline $\mathrm{GO}$ & $10,844(46.04 \%)$ & \\
\hline KEGG & 8,474 (35.98\%) & \\
\hline $\mathrm{COG}$ & 8,565 (36.37\%) & \\
\hline PfAM & $10,821(45.95 \%)$ & \\
\hline SignalP & $1,553(6.59 \%)$ & \\
\hline TmHMM & $3,227(13.7 \%)$ & \\
\hline \multicolumn{3}{|l|}{ (E) SPECIES COMPARISON } \\
\hline Secondary metabolite pathways(KEGG) & 182 & \\
\hline Fatty acid metabolisms(FATT) & 179 & \\
\hline Cytochromes(CYP) & 15 & \\
\hline Carbohydrate-Active enZYmes(CAZY) & 221 & \\
\hline
\end{tabular}

the final model were obtained from the Blast2GO method (Götz et al., 2008).

\section{COMPARATIVE GENOME ANALYSIS}

The total genes of Kolbe were subjected to orthologous analysis to observe the insights of protein compositions among other insects in the Coleoptera taxonomical order. Seventeen genomes (including Kolbe) from fifteen families were used 
in the ortholog analysis using the OrthoMCL method (Li et al., 2003) along with three databases, i.e., cytochrome P450 engineering database (CYPED), carbohydrate-active enzymes database (CAZY) and KEGG database, to obtain the functions (Table 1E). The single-copy genes from the given genomes were subjected to Bayesian evolutionary analysis sampling trees (BEAST), phylogenetic tree reconstruction method, to assess evolutionary time and similarity position among the given genomes (Suchard et al., 2018). Furthermore, to determine the gain and loss of the genes in the given genomes, the proteins were subjected to CAFE v3.1 (Han et al., 2013) method.

\section{PRELIMINARY ANALYSIS REPORT}

Initially, the genome size of Kolbe was estimated to be $656.8 \mathrm{MB}$, with 277.7 GB (401X) of short-read sequences (Figure 1A). The 692.7 MB of the representative draft genome was assembled into 224 contigs from $31.1 \mathrm{~GB}$ (45X) of error-corrected long read sequences (Table 1A; Figures 1B,C). The N50 of the assembled genome is $4.9 \mathrm{MB}$ bases, and $344 \mathrm{MB}$ of the assembled contigs were covered by repeats, which are unclassified elements. Totally, 23,551 genes were predicted from the genome with an average size of $8,217.3$ bases, with the BUSCO score for completeness being 99\% (Table 1B,C). A total of 15,667 (66.52\%) genes are known to have homologous sequences in GenBank, and 10,844 (i.e., 46.04\%) genes also have their gene ontology descriptions (Table 1D and Figure 1C). The evolutionary relationship among these genomes was assessed with 218 single-copy genes through phylogenetic tree reconstruction. The genomes were grouped into exact family clans without any distortion. In continuation, the gain and loss among those genomes were also assessed for the Kolbe genome (Figure 1D). Additionally, the cytochrome family genes from tissue-specific, stage-specific, and differential assessments were conducted. Among these, the Halloween family

\section{REFERENCES}

Bao, W., Kojima, K. K., and Kohany, O. (2015). Repbase Update, a database of repetitive elements in eukaryotic genomes. Mobile DNA 6, 11. doi: 10.1186/s13100-015-0041-9

Blanco, E., Parra, G., and Guig,ó, R. (2002). "Using geneid to Identify Genes," in Current Protocols in Bioinformatics (John Wiley and Sons, Inc.).

Bolger, A. M., Lohse, M., and Usadel, B. (2014). Trimmomatic: a flexible trimmer for Illumina sequence data. Bioinformatics 30, 2114-2120. doi: 10.1093/bioinformatics/btu170

Chin, C.-S., Peluso, P., Sedlazeck, F. J., Nattestad, M., Concepcion, G. T., Clum, A., et al. (2016). Phased diploid genome assembly with single-molecule real-time sequencing. Nat. Methods 13, 1050-1054. doi: 10.1038/nmeth.4035

Götz, S., García-Gómez, J. M., Terol, J., Williams, T. D., Nagaraj, S. H., Nueda, M. J., et al. (2008). High-throughput functional annotation and data mining with the Blast2GO suite. Nucleic Acids Res. 36, 3420-3435. doi: 10.1093/nar/gkn176

Haas, B. J., Delcher, A. L., Mount, S. M., Wortman, J. R., Smith, R. K., Hannick, L. I., et al. (2003). Improving the Arabidopsis genome annotation using maximal transcript alignment assemblies. Nucleic Acids Res. 31, 5654-5666. doi: 10.1093/nar/gkg770

Haas, B. J., Salzberg, S. L., Zhu, W., Pertea, M., Allen, J. E., Orvis, J., et al. (2008). Automated eukaryotic gene structure annotation using EVidenceModeler genes were observed to have differential and tissue-specific expression, which is involved in insect hormone biosynthesis (Rewitz et al., 2007). The specific and differential expressions were observed from RNA-Seq, and the detailed expressions are given in the additional file (Additional File 1).

\section{DATA AVAILABILITY STATEMENT}

The complete sequences generated in this study was deposited to the SRA repository under the accession PRJNA648262. The assembled contigs and its annotation files (CDS, gff, repeats, and proteins) are available in figshare: https:/figshare.com/ s/5e095ac1bf7a63411d23) repository with all the annotations details in Readme file.

\section{AUTHOR CONTRIBUTIONS}

JL, MJ, SS, and YS: genome assembly and annotations. MJ, SS, and YS: manuscript preparation. I-WK, MS, M-AK, SK, JH, EC, and UH: sampling and sequencing. JSH: funding and modeling the study. All authors contributed to the article and approved the submitted version.

\section{FUNDING}

This work was carried out with the support of Cooperative Research Program for National Genome Project (Project No. PJ01338401) Rural Development Administration, Republic of Korea.

\section{SUPPLEMENTARY MATERIAL}

The Supplementary Material for this article can be found online at: https://www.frontiersin.org/articles/10.3389/fgene. 2020.593994/full\#supplementary-material

and the Program to Assemble Spliced Alignments. Genome Biol. 9:R7. doi: 10.1186/gb-2008-9-1-r7

Han, M. V., Thomas, G. W. C., Lugo-Martinez, J., and Hahn, M. W. (2013). Estimating gene gain and loss rates in the presence of error in genome assembly and annotation using CAFE 3. Mol. Biol. Evol. 30, 1987-1997. doi: $10.1093 / \mathrm{molbev} / \mathrm{mst} 100$

Han, R., Shin, J. T., Kim, J., Choi, Y. S., and Kim, Y. W. (2017). An overview of the South Korean edible insect food industry: challenges and future pricing/promotion strategies. Entomol. Res. 47, 141-151. doi: 10.1111/1748-5967.12230

Kim, T.-K., Yong, I. H., Jang, W. H., Kim, Y.-B., and Choi, Y.-S. (2020). Functional properties of extracted protein from edible insect larvae and their interaction with transglutaminase. Foods 9:591. doi: 10.3390/foods9050591

Le Gall, M., Overson, R., and Cease, A. (2019). A global review on locusts (Orthoptera: Acrididae) and their interactions with livestock grazing practices. Front. Ecol. Evol. 7:263. doi: 10.3389/fevo.2019.00263

Lee, J., Bang, K., Hwang, S., and Cho, S. (2016). cDNA cloning and molecular characterization of a defensin-like antimicrobial peptide from larvae of Protaetia brevitarsis seulensis (Kolbe). Mol. Biol. Rep. 43, 371-379. doi: 10.1007/s11033-016-3967-1

Lee, J., Hwang, I. H., Kim, J. H., Kim, M. A., Hwang, J. S., Kim, Y. H., et al. (2017a). Quinoxaline-, dopamine-, and amino acid-derived metabolites from 
the edible insect Protaetia brevitarsis seulensis. Arch. Pharm. Res. 40, 1064-1070. doi: 10.1007/s12272-017-0942-x

Lee, J., Lee, W., Kim, M. A., Hwang, J. S., Na, M., and Bae, J. S. (2017b). Inhibition of platelet aggregation and thrombosis by indole alkaloids isolated from the edible insect Protaetia brevitarsis seulensis (Kolbe). J. Cell Mol. Med. 21, 1217-1227. doi: 10.1111/jcmm.13055

Li, F., Zhao, X., Li, M., He, K., Huang, C., Zhou, Y., et al. (2019a). Insect genomes: progress and challenges. Insect Mol. Biol. 28, 739-758. doi: 10.1111/imb. 12599

Li, L., Stoeckert, C. J. Jr., and Roos, D. S. (2003). OrthoMCL: identification of ortholog groups for eukaryotic genomes. Genome Res. 13, 2178-2189. doi: $10.1101 /$ gr.1224503

Li, Y., Fu, T., Geng, L., Shi, Y., Chu, H., Liu, F., et al. (2019b). Protaetia brevitarsis larvae can efficiently convert herbaceous and ligneous plant residues to humic acids. Waste Manag. 83, 79-82. doi: 10.1016/j.wasman.2018. 11.010

Raheem, D., Raposo, A., Oluwole, O. B., Nieuwland, M., Saraiva, A., and Carrascosa, C. (2019). Entomophagy: nutritional, ecological, safety and legislation aspects. Food Res. Int. 126:108672. doi: 10.1016/j.foodres.2019.108672

Rewitz, K. F., O'connor, M. B., and Gilbert, L. I. (2007). Molecular evolution of the insect Halloween family of cytochrome P450s: phylogeny, gene organization and functional conservation. Insect Biochem. Mol. Biol. 37, 741-753. doi: 10.1016/j.ibmb.2007. 02.012

Shin, G.-H., Shin, Y., Jung, M., Hong, J.-M., Lee, S., Subramaniyam, S., et al. (2018). First draft genome for red sea bream of family sparidae. Front. Genet. 9:643. doi: 10.3389/fgene.2018.00643

Slater, G. S. C., and Birney, E. (2005). Automated generation of heuristics for biological sequence comparison. BMC Bioinform. 6:31. doi: 10.1186/1471-2105-6-31
Stanke, M., Schöffmann, O., Morgenstern, B., and Waack, S. (2006). Gene prediction in eukaryotes with a generalized hidden Markov model that uses hints from external sources. BMC Bioinform. 7:62. doi: 10.1186/1471-2105-7-62

Suchard, M. A., Lemey, P., Baele, G., Ayres, D. L., Drummond, A. J., and Rambaut, A. (2018). Bayesian phylogenetic and phylodynamic data integration using BEAST 1.10. Virus Evol. 4:vey016. doi: 10.1093/ve/vey016

Trapnell, C., Roberts, A., Goff, L., Pertea, G., Kim, D., Kelley, D. R., et al. (2012). Differential gene and transcript expression analysis of RNA-seq experiments with TopHat and Cufflinks. Nat. Protoc. 7, 562. doi: 10.1038/nprot.2012.016

Waterhouse, R. M., Seppey, M., Simão, F. A., Manni, M., Ioannidis, P., Klioutchnikov, G., et al. (2017). BUSCO applications from quality assessments to gene prediction and phylogenomics. Mol. Biol. Evol. 35, 543-548. doi: $10.1093 / \mathrm{molbev} / \mathrm{msx} 319$

Yin, S., Li, G., Liu, M., Wen, C., and Zhao, Y. (2018). Biochemical responses of the Protaetia brevitarsis Lewis larvae to subchronic copper exposure. Environ. Sci. Pollut. Res. 25, 18570-18578. doi: 10.1007/s11356-018-2031-1

Conflict of Interest: MJ, SS, and YS were employed by the company Insilicogen Inc.

The remaining authors declare that the research was conducted in the absence of any commercial or financial relationships that could be construed as a potential conflict of interest.

Copyright $\odot 2021$ Lee, Jung, Shin, Subramaniyam, Kim, Seo, Kim, Kim, Hwang, Choi, Hwang and Hwang. This is an open-access article distributed under the terms of the Creative Commons Attribution License (CC BY). The use, distribution or reproduction in other forums is permitted, provided the original author(s) and the copyright owner(s) are credited and that the original publication in this journal is cited, in accordance with accepted academic practice. No use, distribution or reproduction is permitted which does not comply with these terms. 